\title{
The population of planetary nebulae near the Galactic Centre: chemical abundances
}

\author{
M. Mollá ${ }^{1}$, O. Cavichia ${ }^{2}$, R. D. D. Costa ${ }^{3}$ and W. J. Maciel ${ }^{3}$ \\ ${ }^{1}$ CIEMAT, Avda. Complutense 40, 28040 Madrid, Spain \\ email: mercedes.molla@ciemat.es \\ ${ }^{2}$ IFQ, Universidade Federal de Itajubá, Av. BPS, 1303, 37500-903, Itajubá-MG, Brazil \\ ${ }^{3}$ IAG, Universidade de Sâo Paulo, 05508-900, Sâo Paulo-SP, Brazil
}

\begin{abstract}
In this work, we report physical parameters and abundances derived for a sample of 15 high extinction planetary nebulae located in the inner $2^{\circ}$ of the Galactic bulge, based on low dispersion spectroscopy secured at the SOAR telescope using the Goodman spectrograph. The new data allow us to extend our database including older, weaker objects that are at the faint end of the planetary nebulae luminosity function. The data provide chemical compositions for PNe located in this region of the bulge to explore the chemical enrichment history of the central region of the Galactic bulge. The results show that the abundances of our sample are skewed to higher metallicities than previous data in the outer regions of the bulge. This can indicate a faster chemical enrichment taking place at the Galactic centre.
\end{abstract}

\section{Introduction}

Planetary nebulae (PNe) constitute an important tool to study the chemical evolution of the Milky Way and other galaxies, probing the nucleosynthesis processes, abundance gradients and the chemical enrichment of the interstellar medium. In particular, Galactic bulge PNe (GBPNe) have been extensively used in the literature to study the chemical properties of this Galactic structure. However, the presently available GBPNe chemical composition studies are strongly biased, since they were focused on brighter objects, predominantly located in Galactic regions of low interstellar reddening. The main objective of this work is to derive new physical parameters and chemical abundances of a sample of 15 high extinction planetary nebulae $(\mathrm{PNe})$ located in the inner $2^{\circ}$ of the Galactic bulge, based on low dispersion spectroscopy secured at the $4.1 \mathrm{~m}$ SOAR telescope.

\section{Sample and Observations}

The observed sample is from Jacoby and Van de Steene (2004) and the MASH catalog (Parker et al. 2006). They were observed with the 4.1m SOAR telescope located in Chile (optical spectra) and the $1.6 \mathrm{~m} \mathrm{LNA} /$ Brazil telescope (NIR espectra). The criteria for the galactic coordinates are: $|\ell|<2^{\circ}$ and $|b|<2^{\circ}$. Also, diameters smaller than $12^{\prime \prime}$ and fluxes at $5 \mathrm{GHz}$ lower than $100 \mathrm{mJy}$ were required. The optical spectra of the GBPNe near the Galactic Centre (GC) suffer for high-level of extinction caused by the material near the Galactic plane and also in the central regions of the Galaxy. As a result, important diagnostic lines as [O III] $\lambda 4363 \AA$ and [N II] $\lambda 5755 \AA$ do not have enough $\mathrm{S} / \mathrm{N}$ ratio to obtain the electron temperature from the temperature diagnostic diagrams. Other important temperature-sensitive lines are those from $S^{+3}$. The [S III] auroral line at $\lambda 6312$ $\AA$ is present in most of our optical spectra. We performed near infrared (NIR) observations at the Observatório Pico dos Dias (OPD) of National Laboratory for Astrophysics (LNA, Brazil) with the $1.6 \mathrm{~m}$ Perkin-Elmer telescope. These observations allowed us to 

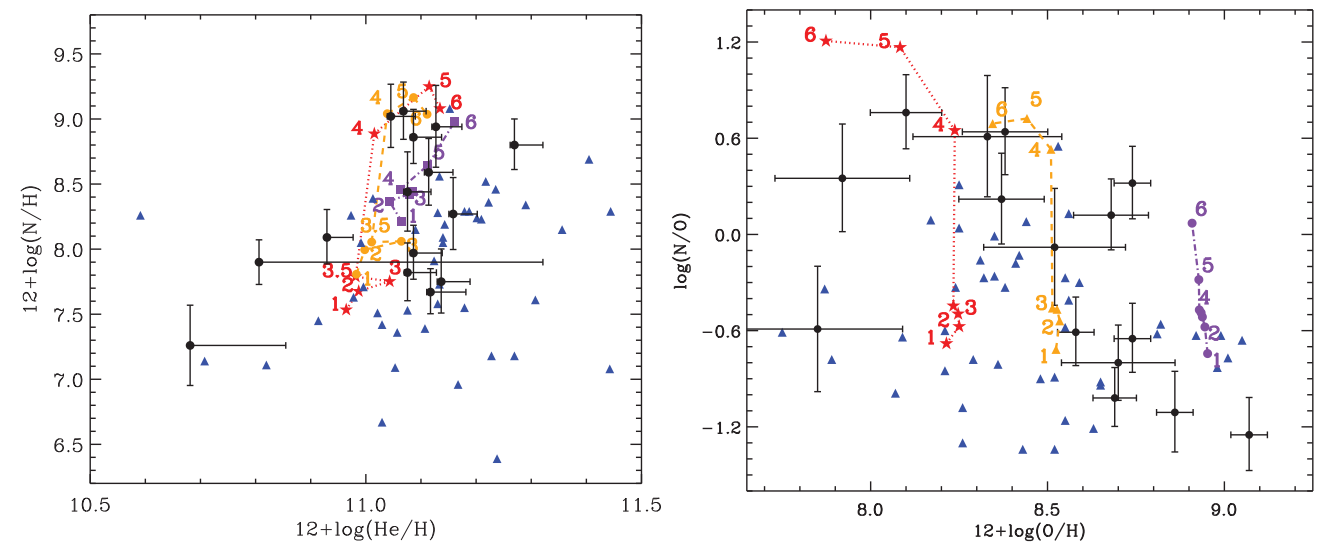

Figure 1. Abundance ratios as a function of $12+\log (\mathrm{He} / \mathrm{H})$ and $12+\log (\mathrm{O} / \mathrm{H})$. Filled circles with error bars are data from present work, while filled blue triangles are the PNe from CCM10 (outer bulge sample). The symbols with numbers represent the results of the models from Karakas (2010) for a given value of $Z$ : Red stars joined by dotted line for $Z=0.004$, yellow triangles joined by dashed line for $Z=0.008$ and purple circles joined by dash-dotted line for $Z=0.02$. The numbers give the initial masses of the individual models in $\mathrm{M} \odot$ units.

observe the [S III] lines at $\lambda \lambda 9069$ and $9532 \AA$, which are suitable to derive the electron temperatures taking into account the high extinction in the visible range. Te[O III] were derived from Te[S III] using the relation provided by Henry et al. (2004). He I emissivities are from Porter et al. (2013). These emissivities include collisional correction. He I abundances are calculated from the flux-weighted mean of the lines 4471, 5876 and $6678 \AA$ A. Elemental abundances for N, O, S and Ar were calculated using the ICFs from Delgado-Inglada et al. (2014). Only in the case of helium we opted not to use any ICF.

\section{Results and Conclusions}

We present the abundances for 15 PNe located near the GC, compared with the data from Cavichia et al. (2010) -hereafter CCM10- (outer bulge sample). The abundance ratios as a function of $12+\log (\mathrm{He} / \mathrm{H})$ and $12+\log (\mathrm{O} / \mathrm{H})$ are shown in Fig. 1.

The abundances of our sample are in agreement with AGB evolution models for masses $<4 \mathrm{M} \odot$. The results show that the abundances in the GBPNe near the GC are skewed to higher metallicities than previous data in the outer regions of the bulge. This can indicate a faster chemical enrichment taking place at the GC. More information can be seen at Cavichia et al.(2017).

\section{References}

Cavichia, O., Costa, R. D. D., \& Maciel, W. J. 2010, Rev. Mex. A\&GA, 46, 159

Cavichia, O., Costa, R. D. D., Maciel, W. J., \& Mollá, M. 2017, MNRAS (submitted)

Delgado-Inglada, G., Morisset, C., \& Stasińska, G. 2014, MNRAS, 440, 536

Henry, R. B. C., Kwitter, K. B., \& Balick, B. 2004, ApJ, 127, 2284

Jacoby, G. H. \& Van de Steene, G. 2004, $A \mathscr{E} A$, 419, 563

Karakas, A. I. 2010, MNRAS, 403, 1413

Parker, Q. A., Acker, A., Frew, D. J., et al. 2006, MNRAS, 373, 79

Porter, R. L., Ferland, G. J., Storey, P. J., \& Detisch, M. J. 2013, MNRAS, 433, L89 